\title{
Detection of Candida Species in the Diabetes Mellitus Patient
}

\author{
Maha M. Rasheed ${ }^{1 *}$, Luma T. Ahmed ${ }^{2}$ and Ahmed M. Athab ${ }^{2}$ \\ ${ }^{1}$ Department of Microbiology, ${ }^{2}$ Department of Pediatrics Medicine, Collage of Medicine, \\ University of Diyala, Iraq \\ *Corresponding author
}

\begin{tabular}{|l|}
\hline Ke y w o r d s \\
$\begin{array}{l}\text { Candida } \text { spp, } \\
\text { Candida albicans \& } \\
\text { diabetes mellitus }\end{array}$ \\
\hline Article Info \\
\hline $\begin{array}{l}\text { Accepted: } \\
10 \text { October } 2018 \\
\text { Available Online: } \\
10 \text { November } 2018\end{array}$ \\
\hline
\end{tabular}

A B S T R A C T

Yeasts of the genus Candida have high genetic variability and are the most common opportunistic pathogenic, which isolated from clinical samples that cause many problems to human, frequently isolated from patient which had diabetes mellitus. The aims of study were Detection and isolation and identification of Candida spp. in Diyala province by routine laboratory procedures. A total of 100 cases between 20 and 90 years old, this study include patients have symptom of oral candidiasis in patient with diabetes mellitus, over a one month period (from December 2017 to February 2018), in Dialay Teaching Hospital. The oral swabs were for cultures on two media, the first media was used for primary isolation which was Sabouraud's dextrose agar (SDA) media and the second was to differentiate Candida spp. according to their colours and also germ tube used for identification of $C$. albicans. Results of this study presented that the highest infection of the Candida spp was accounted for $C$. albicans $31(55.35 \%)$ from the $56(56 \%)$ positive cultures, while other species were as follows: appear Candida glabrata (12) (26.88\%), Candida parapsilosis (6) (13.44\%), Candida krusi (4) (8.96\%), Candida tropical (3) $(6.72 \%)$. Through the analysis of data that collected from patients, there were nonsignificant, relationships were found between Candida infection and age group, gender, smoking type of diabetic. But there was significant, relationship found between candida infection and family history. All data were statistically analyzed depending on SPSS (Statistical Package for Social Science) version 18 (2009) C. albicans is the most common isolated among the total Candida species, and Candida glabrata was the most frequent non-albicans species. In this study we found that patients with type II diabetes mellitus were more infected by Candida albican than type I.

\section{Introduction}

Candida infections are one of the most commonly occurring fungal infections in humans (Kumar et al., 2005). Affecting mucous membrane, skin, nails and internal organs of the body, it additionally a typical opportunist infections in immune compromised patients (Makwana et al., 2012;
Hasan and Al-Jubouri, 2015). Candida species belong to the natural micro biota of an individual's mucosal oral cavity, gastrointestinal tract and vagina (Shao et al., 2007). Although Candida albicans is considered the main agent of candidiasis and to be the most frequently isolated from oral cavity, but in recent two decades there has been important increase of other non-Candida 
albicans species such as Candida glabrata, Candida tropicalis, Candida krusei, Candida parapsilosis and Candida dubliniensisas a result of a different factors like immunesuppressants and prolonged use of broad spectrum antibiotics and antifungal drugs (Martins et al., 2014; Patil et al., 2015; Jain et al., 2016). But the majority of fungal infections in humans are caused by the species C. albicans and C. glabrata.

The prevalence rates of $C$. albicans and $C$. glabrata infections are approximately $70 \%$ and $15 \%$, respectively (Kolaczkowski et al., 2010; Benedetti et al., 2016). Diabetes mellitus is a common and growing global health problem which causes several complications. Periodontal diseases are considered the sixth complication of this disease. Diabetics have an increased predisposition to the manifestations of oral diseases like candidiasis. Diabetes mellitus is the most common endocrine metabolic disorder (Lotfi-Kamran et al., 2009). Nearly $85-90 \%$ of diabetic patients are diagnosed with type II diabetes (resulting from insulin resistance) in these patients, salivary dysfunctions like dry mouth, reduced salivary function, lichen, tooth decay, and periodontal diseases are common (Ship, 2003; Vijan, 2010).

Candida infections are chronic opportunistic infections related to diabetic patients. The presence of Candida spp. in oral cavities of diabetics varies between (50-80\%), (Willis et al., 2000; Khosravi et al., 2008; Melton et al., 2010). Oral candidiasis is a common profiteering infection of the oral cavity caused by an overgrowth of Candida species particularly Candida albicans (Guggenheimer et al., 2000). This infection is usually accompanied by various symptoms including burning, painful sensation, change of taste, reduced saliva secretion and swallowing difficulty, but it can be also asymptomatic
(Nikolic et al., 2016). Among the explanations creating diabetic patients additional susceptible to oral candidiasis (Ship, 2003; Vijan, 2010). Are high levels of salivary glucose, impaired chemotaxis, low secretion of saliva and defect of phagocytosis because of polymorph nuclear white blood cell deficiency (Mohammadi et al., 2016). The attachment of $C$. albicans to the crystalline hydroxyapatite produces collagen lytic protein that will increase crystal solubility and consumes chemical element of dentin albuminoid in diabetes mellitus patients (Mohammadi et al., 2016).

\section{Materials and Methods}

One hundred swabs samples had been collected from patients that have symptom of oral candidiasis from patients with diabetes mellitus. All the collected samples were inoculated directly, on Sabouraud dextrose agar (SDA) containing Chloramphenicol. Than incubated for $48 \mathrm{hrs}$. Each sample of them we had made slide and stain with Lactophenol cotton blue stain for examination of Candida spp.

Than we used germ tube (GT) test it's used to detection of Candida albicans. It is Rapid diagnostic differentiates $C$. albicans from other species. In small micro centrifuge tube One ml of serum was added by using a Pasteur pipette, colony of yeast was transport by sterile wire loop and emulsified it in the serum. We incubated at $37^{\circ}$ for $2-3$ hours but no longer after we mixed than the a drop of the serum was transferred to a slide for examination, cover slip was added and examined microscopically using (40X) objective (Bhavan et al., 2010).

And the only positive samples were cultured on CHROM Agar (CAC). Candida was resuscitated by inoculating a loop full of culture from Sabouraud Dextrose Agar into 
CHROM agar media by streaking a loop full of culture and incubated at $37^{\circ}$ for 72 hours. After 72-96 hours of incubation, the Candida colonies were initially identified by colonial color when compared with standard color photographs supplied by the manufacturer and also presented (Mahmoudabadi et al., 2000).

\section{Results and Discussion}

\section{Culture media}

After the incubation of culture for $48 \mathrm{hrs}$ the morphological feature of culture on (SDA) medium was the most of culture had heavy growth, and the appearances of $C$. albicans colonies on Sabouraud dextrose agar were special it was singular and rounded and the color of the colonies were off-white to creamy and the odor of it was a characteristic yeast odor (Figure 1). Through this study from the 100 samples that cultured on (SDA) there were 56 of them gives positive result (Figure 2). The direct examination under light microscope was done to determine the shape and size of yeast, lactophenol cotton blue stain examination of $c$. albicans isolates showed spherical to oval cells, with a presence of budding and was much larger than bacterial cells (Figure 3).

\section{Phenotype identification of Candida Spp}

The result of germ tube (GT) formation show that from 56 samples that give positive result on SDA only 31 of them give positive for germ tube which seen as along tube - like projections extending from the yeast cells (Figure 4). And the result of CHROM agar shows that the colours of colonies of Candida spp. was similar to colour that given by protocol, green colonies of $C$. albicans. Blue colonies of $C$. tropicalis, Purple- Pink colored colonies of $C$. krusei, C. glabrata produced cream to white and $C$. parapsilosis produced pinkish to white (Figure 5). The present study shows, difference between Candida spp., 31 $(55.35 \%)$ of the total isolated were identified as C. albicans, 25 (44.64\%) isolates were identified as non-albicans., of which Candida glabrata 12 (26.88\%), Candida parapsilosis 6 (13.44\%), Candida krusi 4 (8.96\%), Candida tropical 3(6.72\%).

In this study show relationship between Candida isolated and genders in this study were displayed as it was non-significant $(\mathrm{P}>0.05)$ as it shown in the (Figure 7).

The females 29 samples $(51.8 \%)$ were more infected by candida than males 27 samples $(48.2 \%)$.

According to the type of diabetes the study show that Type II of diabetes 31 samples (55.4\%) higher than the Type I of diabetes 25 samples (44.6\%). There were non-significant $(\mathrm{P}>0.05)$ differences between type diabetes and Candida spp. as shown in the (Figure 8).

According to this study Candida infection rates were higher in the non-smoking patients than smoking patients. There were nonsignificance $(\mathrm{P}>0.05)$ relationship between them. In the case of non-smoking 74 samples $(74.00 \%), 41$ samples $(73.2 \%)$ of them give positive candidiasis while in the case of non smoking there were 15 samples (26.8\%) give positive candidiasis all the result show in the (Figure 9).

Candida infection rate was higher in patient who none have history family of diabetes mellitus 51samples (51\%) in compared with patient that have diabetes in their family history 49samples $(49.00 \%)$. There were high significant $(\mathrm{P}>0.05)$ differences between history family and Candida spp.

In this study, there have been (100) patients from each gender suffered from signs and symptoms of oral candidiasis. 
Fig.1 Colonies of Candida spp. Cultured on SDA at $37^{\circ} \mathrm{c}$ for $48 \mathrm{Hrs}(40 \mathrm{X})$

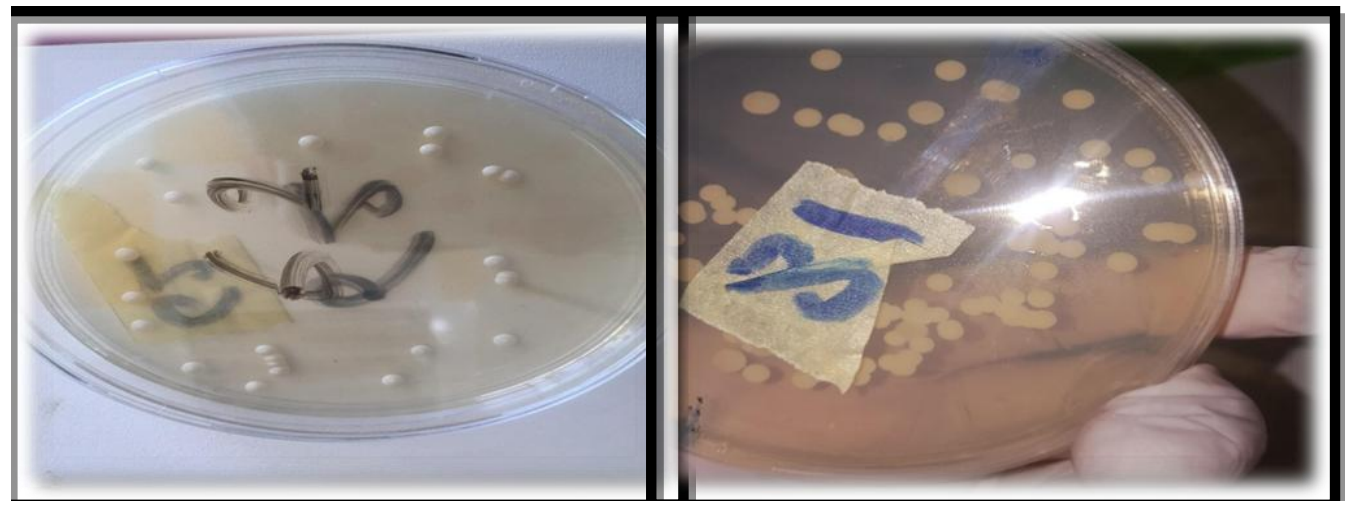

Fig.2 Infections rates Candida spp. among diabetes mellitus patient

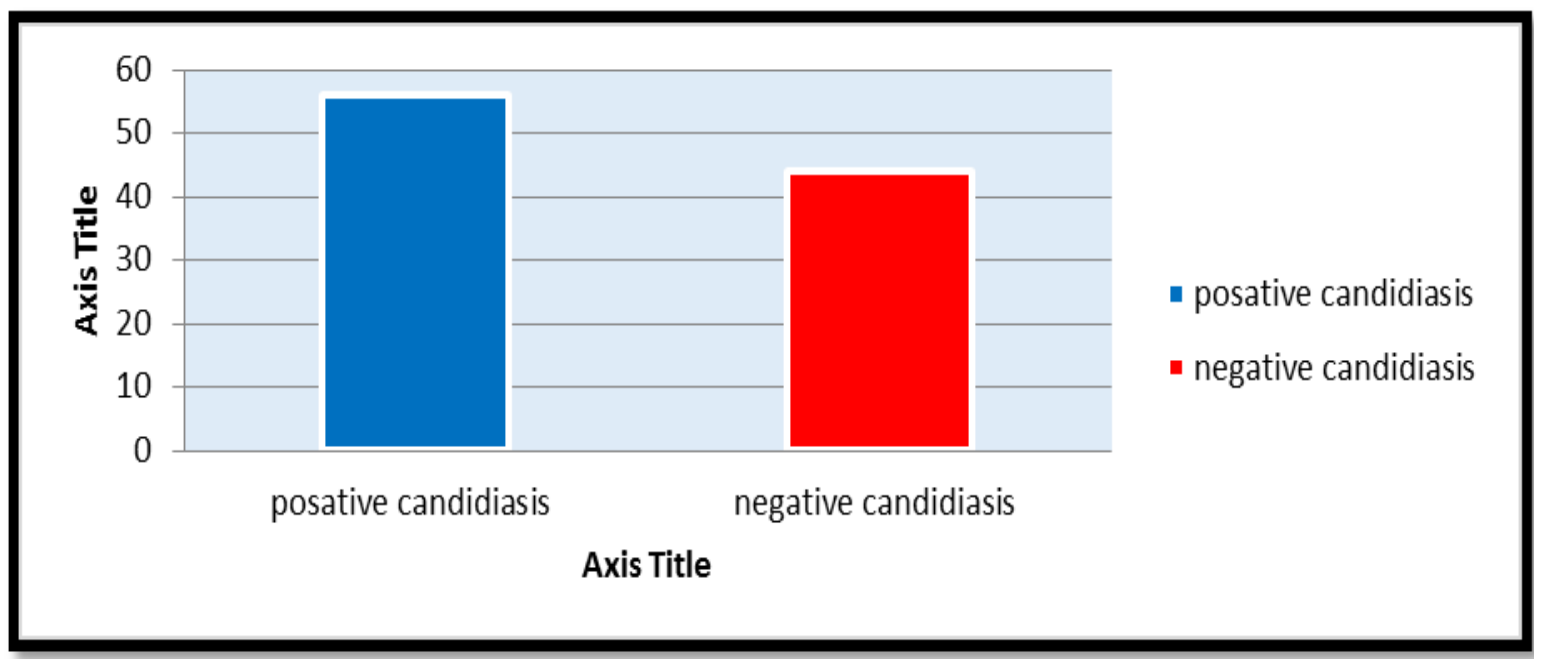

Fig.3 Candida spp. stained with Lactophenol cotton blue (40X)

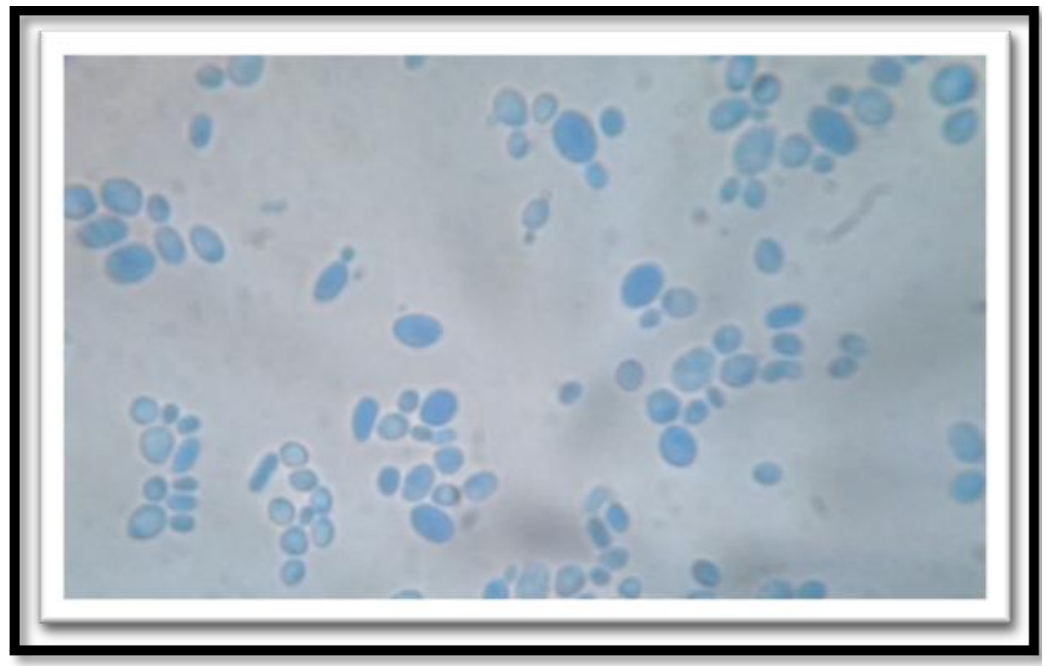


Fig.4 Germ tube formation by C. albicans (40X)

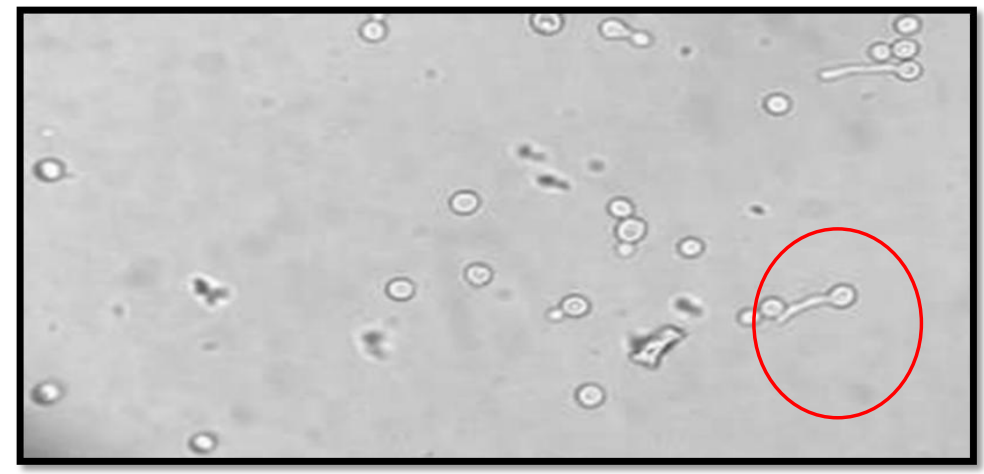

Fig.5 Colonies of Candida spp C. albicans, C. parapsilosis, C. krusei: C. glabrata and C. tropicalis cultured on CHROM agar candida at $37 \mathrm{C}$ for $48 \mathrm{hrs}$ appeared different colors

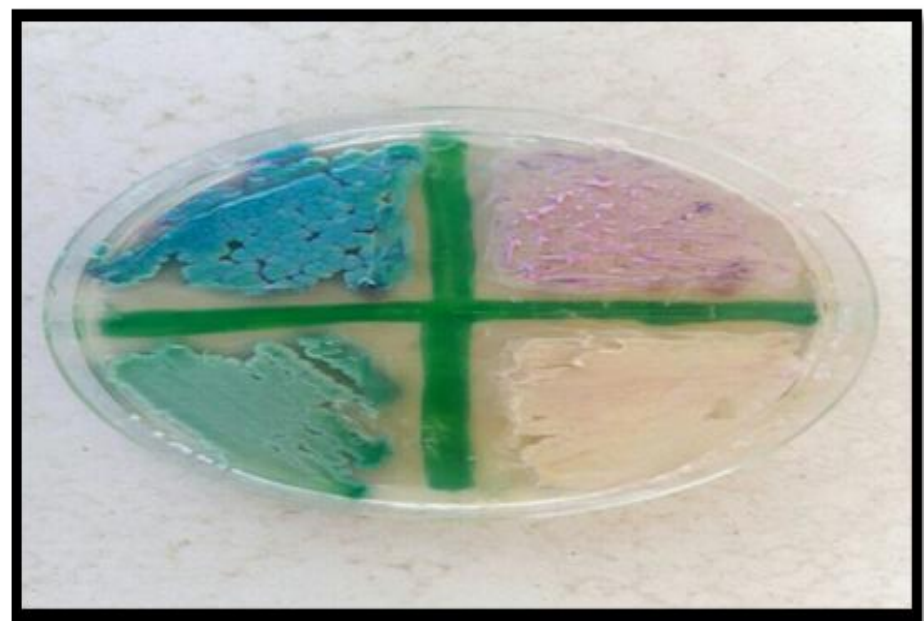

Fig.6 Candida spp. rate isolated from diabetes mellitus patients

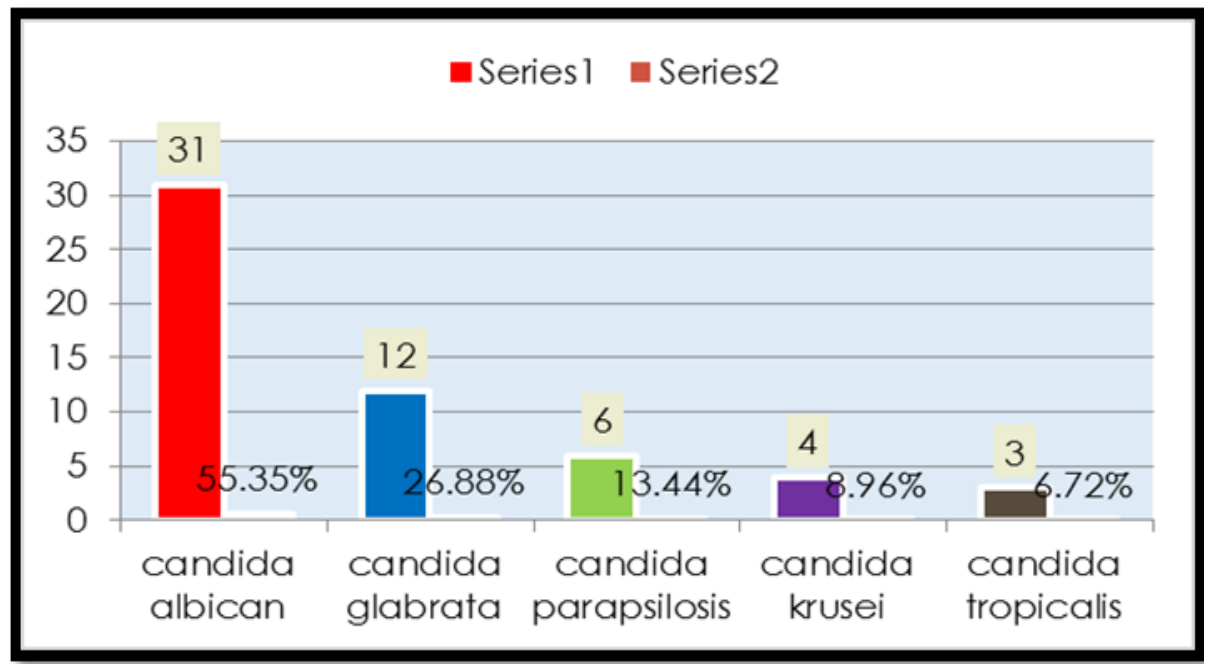


Fig.7 Candida infection rate among patients according to the gender

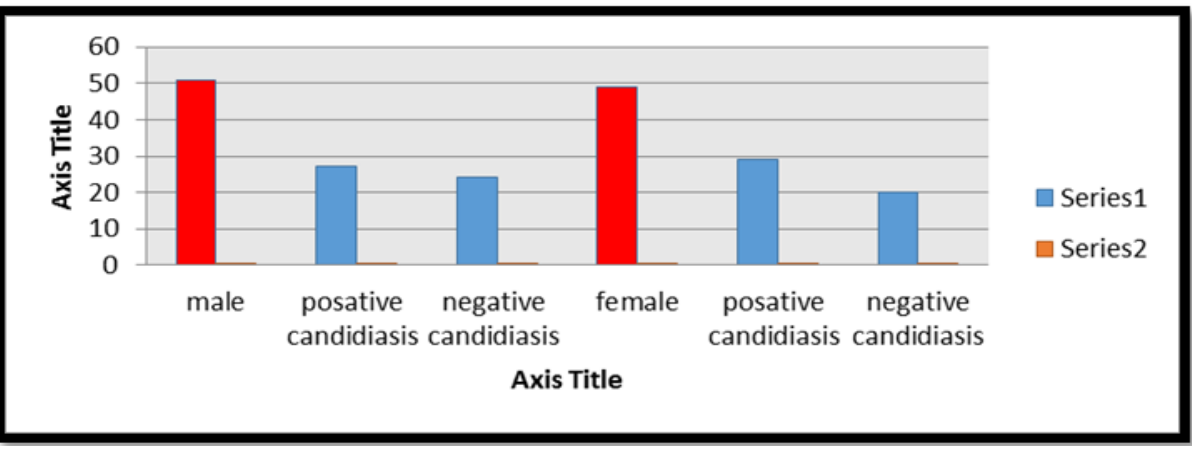

Fig.8 Candida infection rate among patients according to the type diabetes

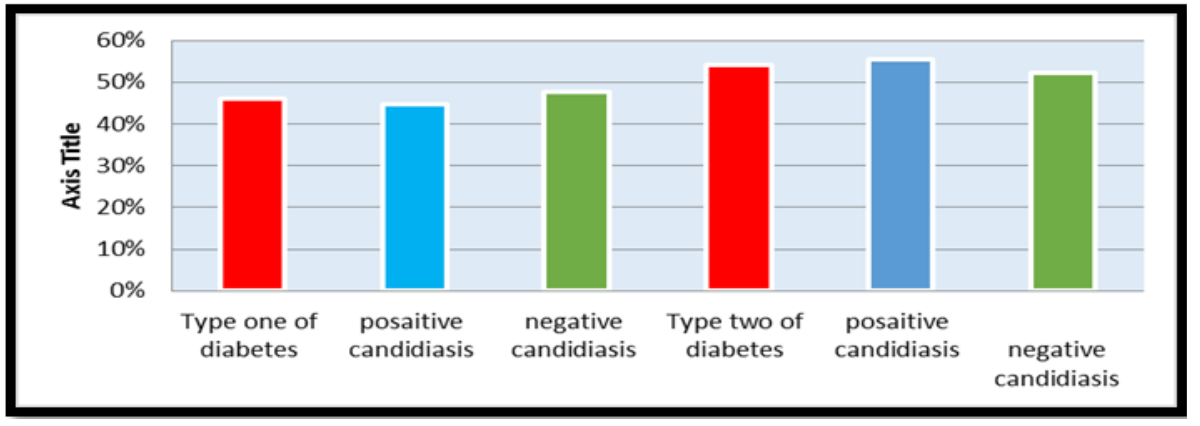

Fig.9 Candida infection rate among patients according to smoking

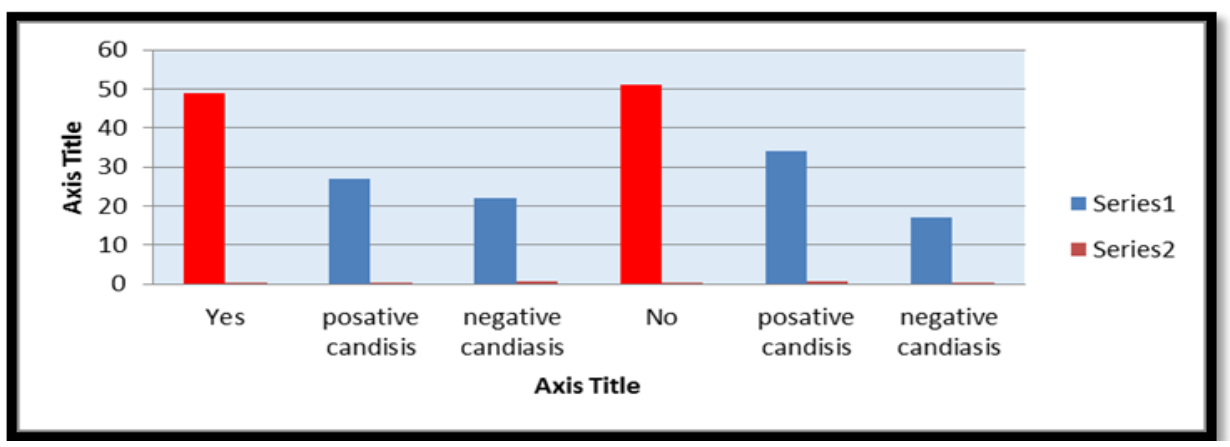

Fig.10 Candida infection rate among patients according to history family

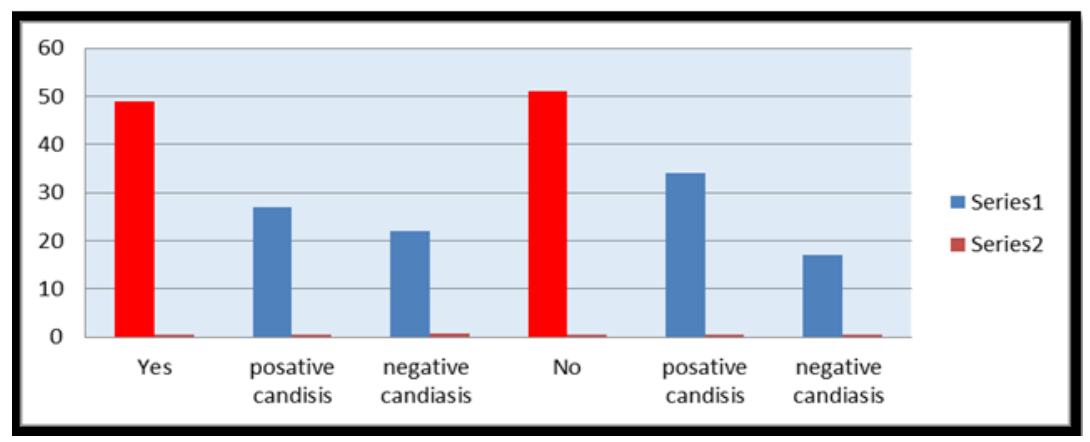


The isolation rates of yeast infection were $(56 \%)$ and the highest infection rates were Candida albican of that $(55.35 \%)$, this study agree with Amin and Kasim and Jasim all found that Candida albican is highest infection (Amin et al., 2014; Kasim and Yehia, 2006; Jasim et al., 2016).

Candida spp. was phenotype known depending on the morphological features include germ tube, and chromogenic agar candida (CAC). The germ tubes were formed with more than two hours of incubation and this can be a singular identification characteristic of $C$. albicans differentiates it from other fungi. Other sort of yeast-like isolated gave negative result to germ tube.

The result of germ tube agreed with (Jasim et al., 2016; Sudarsanam et al., 2015; ALAttraqchi et al., 2017; Matare et al., 2017) the germ tube was formed within two hours of incubation and it is a unique diagnostic characteristic of $C$. albicans that differentiates it from other species. Formation of germ tube is associated with increased synthesis of protein and ribonucleic acid. And it were solutions contains tryptic soy broth and fetal bovine serum, essential nutrients for protein synthesis.

It is lyophilized for stability. Germ tube is one of the virulence factors of Candida albicans. This is a rapid test for the hypothetical identification of C. albicans (Matare et al., 2017).

The results of chromogenic agar candida $(\mathrm{CAC})$ were in agreement with Mohammdi et al., (2016) he found in his study that Candida albicanwas higher $34(36.2 \%)$ and were $C$. glabrata, $(0 \%)$ C. parapsilosis, $3(5.2 \%) C$. krusei2 (3.4\%) were C. tropicalis. And it is in agreement with Suarez et al., (2013) in the Colombia that he had found $(29.0 \%) C$. albicanand $5(9.0 \%)$ C. parapsilosis, (0\%) were $C$. tropicalis and it is also agreed with Benedetti et al., (2016) that he had found $C$. albicans (39) C. parapsilosis (10) C. glabrata (1) C. tropicalis (5). And agree with de la Rosa-García et al., (2013) in his study on Oral colonization and infection by Candida spp. In diabetic and non-diabetic patients with chronic kidney disease on dialysis.it was found that $22(39.3 \%)$ C. albicans, C. globate $7(12.5 \%), 6(7.5 \%)$ C. tropicalis $6(10.7 \%)$ and $(0 \%)$ C. parapsilosis,

Chromogenic media were effective for isolating fungi and identifying Candida species, especially $C$. albicans, another advantage of these chromogenic media was their ease of use in the identification of nonC. albicans Candida species, 24-48 hrs earlier than traditional media. However, as expected and observed, performances of various chromogenic media differ from each other.

They also differ over time, because they are subject to continuous development (Davis and Rabinowitz, 2007) Candida spp, usually found in humans, can be isolated in about $50 \%$ of healthy population without clinical signs of infection In the case of diabetics, this prevalence is even higher (Davis and Rabinowitz, 2007).

The distribution of infection showed that males had 27 samples (48.2\%) while females had 29 samples $(51.8 \%)$. These results were in agreement with Matic et al., (2015) at Siberia males 14 (40\%) and females 16 (60\%) in group B that include diabetic patients with good metabolic, and it was agreed with AlAttas and Amro, (2010) in Saudi Arabia, males were (41.35) and females (58.7\%) and it was non- significant.

It was non-significant between type of diabetes and candidiasis, and through this study Type II 31 samples (55.4\%) were higher than type I 25 samples (44.6\%) and the 
results similar to results of Al-Attas and Amro, (2010) it found that type II 17 (73.9\%) were higher than type I 14 (63.6\%), and it agreed with Amin et al., (2014). Also, agreed with Mohammadi et al., (2016) and both found that higher infection in type II.

This higher rang of candida prevalence in diabetes mellitus could also be explained by the actual fact that the natural oral flora is modification by the endocrine abnormalities in diabetes mellitus.

The development of candidal formation in diabetic patients is also refer to the larger adherence of fungi to epithelial cells, assist by the increased glucose content within the saliva, genetic susceptibility to infection, altered cellular and humoral immune defense mechanisms, and native factors, as well as poor blood supply (Bremenkamp et al., 2011).

Candida infection rates were higher in nonsmoking patient than smoking patients. In the case of nonsmoking there were 41 samples $(73.2 \%)$ given positive for candidiasis and 15 samples $(26.8 \%)$ that give positive for candidiasis in smoker patients.

This study was in agreement with Al-Attas and Amro et al., (2010), In the case of nonsmoking there were $(89.1 \%)$ given positive for candidiasis and $(10.1 \%)$ that give positive for candidiasis in smoker patient, and it agree with Matić et al., (2015) it also there was nonsignificant with smoker patient.

While, it disagreement with Abu-Elteen et al., (2006) who mentioned that smoking people is more infected with candida spp.

There were high significant ( $\mathrm{P}$ 0.028) differences between history family and Candida spp. Candida infection rates were higher in patients who have no history family of diabetes mellitus 34 samples (60.7\%) of them given positive for candidiasis.
While, that have history family among them there were 22 samples (39.3\%) given positive result to candidiasis. this disagreement with Sousa et al., (2011) in here study Clinical study of the oral manifestations and related factors in type 2 diabetics patients it found that have history family $66(68.8 \%)$ more than that have no history family $30(31.1 \%)$.

\section{References}

Abu-Elteen, K. H., Hamad, M. A., and Salah, S. A. (2006). Prevalence of oral Candida infections in diabetic patients. Bahrain Medical Bulletin., 28(1): 1-8.

Al-Attas, S. A., and Amro, S. O. (2010). Candidal colonization, strain diversity, and antifungal susceptibility among adult diabetic patients. Annals of Saudi medicine, 30(2): 101.

AL-Attraqchi, A. A., Hassan, J. S., Dawood, H. N., and Hadab, M. A. (2017). Molecular and conventional methods for detection of Candida species isolated from a sample of immunocompromised Iraqi patients with pulmonary symptoms. Journal of Contemporary Medical Sciences, 3(10): 229-233.

Amin, A. M., Sadiq, N., and Saeed, C. H. (2014). Isolation of Candida albicans from oral cavity of type II diabetic subjects and its relationship to total and differential white blood cell count. Zanco J Med Sci, 18(3): 833-838.

Benedetti, V. P., Savi, D. C., Aluizio, R., Adamoski, D., Kava-Cordeiro, V., GalliTerasawa, L. V., and Glienke, C. (2016). Analysis of the genetic diversity of Candida isolates obtained from diabetic patients and kidney transplant recipients. Memórias do Instituto Oswaldo Cruz., 111(7): 417-422.

Bhavan, P.S., Rajkumar, R., Radhakrishnan, S., Seenivasan, C. and Kannan, S. (2010). Culture and identification of Candida albicans from vaginal ulcer and separation of enolase on SDS-PAGE. Inter. J. Bio., 2(1): 84-93. 
Bishara, W., Sikora, U., Mudanyali, O., Su, T. W., Yaglidere, O., Luckhart, S., and Ozcan, A. (2011). Holographic pixel super-resolution in portable lensless onchip microscopy using a fiber-optic array. Lab on a Chip, 11(7), 1276-1279.

Bremenkamp, R., Caris, A. R., Jorge, A. O. C., Back-Brito, G. N., Mota, A. J., Balducci, I., and Koga-Ito, C. Y. (2011). Prevalence and antifungal resistance profile of Candida spp. oral isolates from patients with type 1 and 2 diabetes mellitus. Archives of oral biology, 56(6), 549-555.

Davis, P. J., and Rabinowitz, P. (2007). Methods of numerical integration. Courier Corporation.

De la Rosa-García, E., Miramontes-Zapata, M., Sánchez-Vargas, L. O., and MondragónPadilla, A. (2013). Oral colonisation and infection by Candida sp. in diabetic and non-diabetic patients with chronic kidney disease on dialysis., Nefrologia: 33(6).

Guggenheimer, J., Moore, P. A., Rossie, K., Myers; D., Mongelluzzo, M. B., Block, H. M., and Orchard, T. (2000). Insulindependent diabetes mellitus and oral soft tissue pathologies. II. Prevalence and characteristics of Candida and candidal lesions. Oral Surgery, Oral Medicine, Oral Pathology, Oral Radiology, and Endodontology., 89(5): 570-576.

Hasan, A. M., and Al-Jubouri, M. H. (2015). Isolation of Candida Spp. from Patients with Different Types of Leukemia Who Suffered Oral Candidiasis Due to Their Weekend Immune System. J Pharm Chem Biol Sci, 3(1): 79-83.

Jain, M., Shah, R., Chandolia, B., Mathur, A., Chauhan, Y., Chawda, J., and Bhagalia, S. (2016). The oral carriage of Candida in oral cancer patients of Indian origin undergoing radiotherapy and/or chemotherapy. Journal of clinical and diagnostic research: JCDR., 10(2): ZC17.

Jasim, S. T., Flayyih, M. T., and Hassan, A. A. (2016). Isolation and identification of Candida spp. from different clinical specimens and study the virulence factors.
World J Pharm Pharmaceut Sci., 5(7): 121-37.

Kasim, Z. S., and Yehia, M. M. (2006). Oral candidal colonization in immune compromised patients. Iraqi Journal of Pharmacy, 13(2): 17-26.

Khosravi, A. R., Yarahmadi, S., Baiat, M., Shokri, H., and Pourkabireh, M. (2008). Factors affecting the prevalence of yeasts in the oral cavity of patients with diabetes mellitus. Journal de Mycologie Médicale/ Journal of Medical Mycology., 18(2): 8388.

Kolaczkowski, M., Kolaczkowska, A., Środa, K., Ramalhete, C., Michalak, K., Mulhovo, S., and Ferreira, M. J. U. (2010). Substrates and modulators of the multidrug transporter Cdr1p of Candida albicans in antifungal extracts of medicinal plants. Mycoses, 53(4): 305310.

Kumar, B. V., Padshetty, N. S., Bai, K. Y., and Rao, M. S. (2005). Prevalence of Candida in the oral cavity of diabetic subjects. The Journal of the Association of Physicians of India. 53: 599-602.

Lotfi-Kamran, M. H., Jafari, A. A., Falah-Tafti, A., Tavakoli, E., and Falahzadeh, M. H. (2009). Candida colonization on the denture of diabetic and non-diabetic atients. Dental research journal., 6(1): 23.

Mahmoudabadi, A. Z., Drucker, D. V., Mandall N; O'Brien, K. and Theaker, E. (2000) Isolation and Identification of Candida Species from the Oral Cavity Using CHROM agar Candida, Iranian Biomedical Journal, 4(2-3), 57-61.

Makwana, G. E., Gadhavi, H., and Sinha, M. (2012). Comparison of germ tube production by Candida albicans in various media. NJIRM., 3: 6-8.

Martins N., Ferreira I.C., Barros L., Silva, S., Henriques, M. (2014). "Candidiasis: predisposing factors, prevention, diagnosis and alternative treatment". Mycopathologia., 177 (5-6):223-240.

Matare, T., Nziramasanga, P., Gwanzura, L., and Robertson, V. (2017). Experimental Germ Tube Induction in Candida 
albicans: An Evaluation of the Effect of Sodium Bicarbonate on Morphogenesis and Comparison with Pooled Human Serum. BioMed research international, 2017.

Matić Petrović, S., Cimbaljević, M., Radunović, M., KuzmanovićPfićer, J., Jotić, A., and Pucar, A. (2015). Detection and Sampling Methods for Isolation of Candida spp. From Oral Cavities in Diabetics and NonDiabetics. Brazilian Oral Research., 29(1): 1-7.

Melton, J. J., Redding, S. W., Kirkpatrick, W. R., Reasner, C. A., Ocampo, G. L., Venkatesh, A., and Mealey, B. L. (2010). Recovery of Candida dubliniensis and other Candida species from the oral cavity of subjects with periodontitis who had well- controlled and poorly controlled type 2 diabetes: a pilot study. Special Care in Dentistry., 30(6): 230-234.

Mohammadi, F., Javaheri, M. R., Nekoeian, S., and Dehghan, P. (2016). Identification of Candida species in the oral cavity of diabetic patients. Current medical mycology, 2(2): 1.

Nikolic, M., Smiljkovic, M., Markovic, T., Cirica, A., Glamoclija, J., Markovic, D., and Sokovic, M. (2016). Sensitivity of clinical isolates of Candida to essential oils from Burseraceae family. EXCLI journal., 15: 280.

Patil, S., Rao, R. S., Majumdar, B., and Anil, S. (2015). Clinical appearance of oral
Candida infection and therapeutic strategies. Frontiers in microbiology., 6: 1391.

Shao, L. C., Sheng, C. Q., and Zhang, W. N. (2007). Recent advances in the study of antifungal lead compounds with new chemical scaffolds. Yao xuexuebao= Actapharmaceutica Sinica., 42(11): 11291136

Ship, J. A. (2003). Diabetes and oral health: an overview. The Journal of the American Dental Association, 134: 4S-10S.

Sousa, M. G. D. M., Costa, A. D. L. L. and Roncalli, A. G. (2011). Clinical study of the oral manifestations and related factors in type 2 diabetics patients. Brazilian journal of otorhinolaryngology., 77(2): 145-152.

Sudarsanam, P., Hillary, B., Deepa, D. K., Amin, M. H., Mallesham, B., Reddy, B. M., and Bhargava, S. K. (2015). Highly efficient cerium dioxide nanocube-based catalysts for low temperature diesel soot oxidation: the cooperative effect of cerium-and cobalt-oxides. Catalysis Science \& Technology, 5(7), 3496-3500.

Vijan, S. (2010). Type 2 diabetes. Annals of internal medicine., 152(5): ITC3-1.

Willis, A. M., Coulter, W. A., Hayes, J. R., Bell, P., and Lamey, P. J. (2000). Factors affecting the adhesion of Candida albicans to epithelial cells of insulinusing diabetes mellitus patients. Journal of medical microbiology., 49(3): 291-293.

\section{How to cite this article:}

Maha M. Rasheed, Luma T. Ahmed and Ahmed M. Athab. 2018. Detection of Candida Species in the Diabetes Mellitus Patient. Int.J.Curr.Microbiol.App.Sci. 7(11): 1111-1120. doi: https://doi.org/10.20546/ijcmas.2018.711.129 